\title{
Amebiasis in Four Ball Pythons, Python reginus
}

\author{
Atsuko KOJIMOTO ${ }^{1)}$, Kazuyuki UCHIDA ${ }^{1) *}$, Yoichiro HORII'), Sumiyo OKUMURA ${ }^{3)}$, Ryoji YAMAGUCHI ${ }^{1)}$ and \\ Susumu TATEYAMA ${ }^{1)}$
}

${ }^{1)}$ Departments of Veterinary Pathology and ${ }^{2)}$ Veterinary Internal Medicine, Faculty of Agriculture, Miyazaki University, Miyazaki 8892155, and ${ }^{3)}$ Phoenix Natural Zoological Park, Miyazaki 880-0122, Japan

(Received 22 May 2001/Accepted 3 September 2001)

ABSTRACT. Between September 13th and November 18th in 1999, four ball pythons, Python reginus kept in the same display, showed anorexia and died one after another. At necropsy, all four snakes had severe hemorrhagic colitis. Microscopically, all snakes had severe necrotizing hemorrhagic colitis, in association with ameba-like protozoa. Some of the protozoa had macrophage-like morphology and others formed protozoal cysts with thickened walls. These protozoa were distributed throughout the wall in the large intestine. Based on the pathological findings, these snakes were infested with a member of Entamoeba sp., presumably with infection by Entamoeba invadens, the most prevalent type of reptilian amoebae.

KEY WORDS: ball python, colitis, reptilian amebiasis.

In reptiles kept in zoos, most causes of death and underlying diseases remain unknown $[2,6]$. Reptilian amebiasis, which results from infection by Entamoeba invadens, is known to be pathogenic in snakes and some other reptiles, and is characterized by ulcerative colitis and hepatitis $[4,5]$. In the literature, amebiasis is one of the most common gastrointestinal diseases in snakes, and some biological, epidemiological, and clinical studies on E. invadens have been published [3]. E. invadens is morphologically, or biologically similar to E. histolytica in humans, and its host affinity is already well known. E. invadens infestation is reported to have occurred at large dioramas in the U.S.A. and in other countries [3], while in Japan this disease has not been recognized in snakes. In the present paper, we describe pathological features in examinations of four snakes, ball python, Python reginus, which had been kept in the same display, and died within a short period of time. All of the snakes died from severe colitis which is typical of reptilian amebiasis, presumed to be E. invadens.

Complete necropsies were performed on four ball pythons, Python reginus (Fig. 1), which died between 13 September and 18 November 1999. All of these snakes had been brought to Phoenix Natural Zoological Park in Miyazaki from Hiroshima Municipal Asa Zoological Park, Hiroshima, Japan in 1997, and were exhibited together in the same display. Their clinical signs are summarized in Table 1. Clinically, these snakes showed signs of anorexia, and sometimes regurgitation, for 3 to 30 days before death. The first three snakes died almost simultaneously, and received no medical treatment. The last snake was treated for 26 days with metronidazole ( $100 \mathrm{mg} / \mathrm{kg}$, p.o.) for 5 days and subcutaneous infusions. These snakes were necropsied at the zoo, and samples of the lung, heart, liver, stomach, large and small intestine, and kidneys, were fixed in $10 \%$

\footnotetext{
* Correspondence to: Uchida, K., Department of Veterinary Pathology, Faculty of Agriculture, Miyazaki University, Miyazaki 889-2155, Japan.
}

formalin for further histopathological examinations. The tissues were embedded in paraffin, and $4 \mu \mathrm{m}$ thick sections were stained with hematoxylin-eosin (HE). Some selected sections were also stained with periodic acid-Schiff (PAS) and periodic methenamine silver (PAM). Fresh tissues from case No. 3 were submitted for immunofluorescence assay. Cryostat sections from the large intestines and liver were fixed in cold acetone for $5 \mathrm{~min}$ and were incubated with a monoclonal antibody against $E$. histolytica antibody (1:100, Biogenesis, Poole, U.K.) at $37^{\circ} \mathrm{C}$ for $30 \mathrm{~min}$. Following subsequent incubation with fluorescein-isothiocyanate-conjugated rabbit serum against mouse immunoglobulins (1:40, DAKO-Japan, Kyoto, Japan) at $37^{\circ} \mathrm{C}$ for $30 \mathrm{~min}$, the sections were observed under a fluorescence microscope.

At necropsy, all cases had severely swollen and congested walls of the large intestines, about $10 \mathrm{~cm}$ rostal from the cloaca (Fig. 2a). The mucosal layer was thickened, and covered with a dark-red pseudomembrane consisting of necrotic tissues and fibrinous exudate (Fig. 2b). The livers were markedly congested, and multifocal red spots were distributed. Other visceral organs had no significant gross lesions. Histopathologically, the intestine had severe necrosis with multifocal hemorrhage, resulting in pseudomembrane formation throughout the mucosa. In the submucosa various degrees of diffuse hemorrhage and edema, as well as moderate infiltrations of heterophils, lymphocytes and histiocytes, were observed (Fig. 3). In some areas, the inflammation was seen transmurally. In these lesions, a large number of macrophage-like protozoal organisms were observed, together with many bacterial colonies. The organisms in the intestinal parenchyma, which were thought to be protozoan trophozoites, approximately $10 \mathrm{~nm}$ in diameter, were irregularly round in appearance, with abundant cytoplasm and a central round nucleus (Fig. 4a). In the intestinal lumen, however, they were spheroidal in shape with a thickened cell wall and several rod-shaped nuclei that were thought to be protozoan cysts (Fig. 4b and 4c). These 
Table 1. Clinical features of Ball Pythons examined

\begin{tabular}{ccccclc}
\hline $\begin{array}{c}\text { Case No. } \\
\text { No. }\end{array}$ & $\begin{array}{c}\text { Age } \\
\text { (years) }\end{array}$ & Sex & $\begin{array}{c}\text { Body weight } \\
(\mathrm{g})\end{array}$ & $\begin{array}{c}\text { Length } \\
(\mathrm{cm})\end{array}$ & Clinical signs & Clinical treatments \\
\hline 1 & 5 & $\mathrm{~F}$ & 1450 & 115 & $\begin{array}{l}\text { Anorexia for } 9 \text { days } \\
\text { Anorexia for } 15 \text { days }\end{array}$ & No \\
2 & 5 & $\mathrm{~F}$ & 1600 & 115 & No \\
3 & 3 & $\mathrm{M}$ & 950 & 93 & $\begin{array}{l}\text { Anorexia for 3 days } \\
\text { Anorexia about } \\
\text { for 30 days }\end{array}$ & $\begin{array}{l}\text { Metronidazole }(100 \mathrm{mg} / \mathrm{kg} \\
\text { p.o., } 5 \text { days, }) \text { subcutaneous } \\
\text { infusion (every 3 or } 4 \text { days), } \\
\text { and antibiotics }\end{array}$ \\
\hline
\end{tabular}

organisms were intensely positive for both PAS and PAM stains. Their morphological features were suggestive of the genus Entoamoeba. The liver was severely congested, and had vacuolated cytoplasm of hepatocytes which was indicative of fatty degeneration in all cases. There was a moderate degree of multifocal infiltrations of heterophils surrounding intralesional protozoa. In all snakes, the trophozoite-like organisms of amoeba were found occasionally with or without inflammatory changes. In addition to these changes, mild to moderate degrees of peritonitis, catarrhal gastritis, interstitial nephritis and nephropyelitis, and suppurative pneumonia were observed. The brain in one case (No. 3) examined, exhibited multifocal necrosis with intralesional protozoal organisms. Case No. 4, which was treated with metronidazole, also had generalized edema, which might have been due to the infusion. The immunofluorescence assay revealed ring-like fluorescence-signals, representing protozoan cysts in the intestinal lumen. In the intestinal parenchyma, many randomly distributed, presumably immunopositive trophozoites were observed, although it was hard to distinguish a significant signal from non-specific reactions.

It was concluded that the four ball pythons examined had severe hemorrhagic and necrotic colitis with lesional protozoan organisms, probably a group of Entamoeba sp. Moreover, the protozoan infection was disseminated, as it was observed in almost all of the organs. Donaldson et al. indicated that $E$. invadens is one of the most important infectious agents among snakes [1, 3]. It resembles $E$. histolytica, but can be distinguished by the host species and its temperature tolerance $[1,3]$. The pathological lesions observed in the present ball pythons were almost in conformity with those of $E$. invadens infections described previously in snakes $[1,3]$. Thus, although E. invadens is thought to have been the agent of severe colitis in the ball pythons examined, protozoan isolation and further examinations are required for accurate identification of the species. In an immunohistochemical study, Jakob and Wesemeier [5] successfully used an antibody to E. invadens to prove infestation. In the present study, an immunofluorescence assay using an antibody against E. histolytica was performed, and lesional protozoan cysts showed an immunopositive-reaction. Since there was also a moderate non-specific reaction in the tissues, we could not conclude that the antibody suc- cessfully revealed the species of this amoeba. The immunoperoxidase method using the antibody was unsuccessful because of non-specific reaction.

The biological host for E. invadens is thought to be herbivorous turtles, in which a symbiotic relationship without any pathogenicity may be observed [7]. Turtles may therefore be responsible for spreading of the pathogens to other susceptible reptiles. In the intestine of turtles, amoebic protozoa take nourishment from ingested plants to form cysts, and complete their life cycle without being pathogenic to the host. However, in the intestine of other carnivorous reptiles, the amoeba is unable to take the specific nourishments it requires, and has to invade the intestinal mucosa to survive, resulting in harmful infection of the host [7]. With respect to the present cases, some turtles were introduced to the zoo before the outbreaks of the present snake infection. It is suspected that these turtles were the source of that infection.

Clinically, reptilian amebiasis are treated with the antiprotozoan drug, metronidazole, at a dose of $275 \mathrm{mg} / \mathrm{kg}$ by using a stomach tube. This treatment was remarkably effective to infected snakes when it was used either once or several times [3]. However, for case No. 4 in the present study, treatments using metronidazole $(100 \mathrm{mg} / \mathrm{kg}$ p.o.), were not effective to the infected snake. Post mortem examination of this case revealed the same pathological changes as those observed in the other three snakes, suggesting that the intestinal lesions were already too severe at the time when the metronidazole treatment was started. In order to prevent further infections of other reptiles, it is important that the exhibition cages should be sterilized and animal keepers should be aware of the correct sanitation procedures. Prophylactic treatment of the reptiles with metronidazole is also recommended.

Since there is little detailed information concerning the incidence of reptilian amebiasis in Japan, investigations into the epidemiology of this disease are required. In addition, the development of specific examinations that allow the differentiation between amebiasis and other causative agents is necessary for the successful treatment and prevention of this disease.

\section{REFERENCES}

1. Barrow, J.H. 1960. J. Protozool. 7: 377-383. 


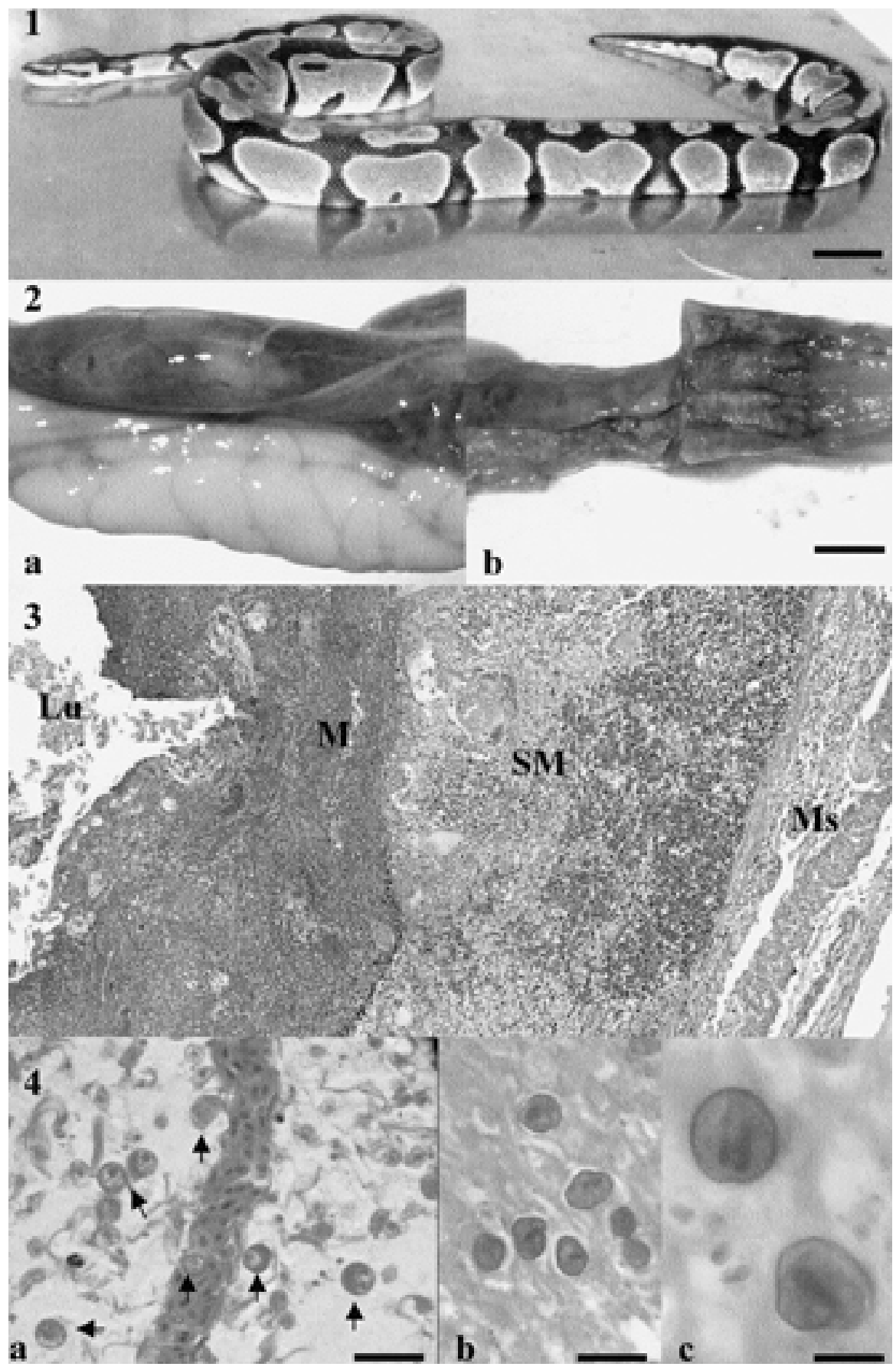

Fig. 1. Ball python, Python reginus, case No. 1. An overview of the ball python at necropsy. Bar=5 $\mathrm{cm}$

Fig. 2. Severe swelling and congestion of the large intestine (a). Pseudomembrane formation on the surface of mucosal layer (b). Case No. 3. Bar $=5 \mathrm{~mm}$.

Fig. 3. The mucosa replaced by pseudomembrane consisting of fibrinous exudate, necrotic mucosal tissues and bacterial colonies. In the submucosal layer, there are severe diffuse necrosis and hemorrhage with moderate infiltration of heterophils. Case No. 2. HE. $\times 20$. Lu: lumen, M: mucosa, SM; submucosa, Ms; muscular layer.

Fig. 4. a: Macrophage-like protozoan organisms thought to be trophozoites (arrows) in the submucosal layer. Case No. 2. HE. Bar $=25 \mu \mathrm{m}$, b: Protozoan cysts within the lumen by PAS stain. Bar $=25 \mu \mathrm{m}, \mathrm{c}$ : High power magnification of protozoan cysts with rod-shaped central and ubiquitous nuclei by PAM stain. Bar $=10 \mu \mathrm{m}$. 
2. Cooper, J.E. 1986. Vet. Rec. 119: 459-460.

3. Donaldson, M. 1975. Am. J. Vet. Res. 36: 807-817.

4. Jacobson, E. 1983. J. Am. Vet. Med. Assoc. 183: 1993-1994.

5. Jakob, W. and Wesemeier, H.H. 1995. J. Comp. Pathol. 112:
$417-421$.

6. Kaneene, J.B. 1985. J. Am. Vet. Med. Assoc. 187: 1332-1333.

7. Meerovitch, E. 1958. Can. J. Zool. 36: 513-523. 